\title{
Crescimento e esporulação de isolados de Verticillium lecanii sob diferentes fatores ambientais
}

\author{
Antonio Carlos Monteiro(1), Claudio Camargo Barbosa( ${ }^{(1)}$, Antônia do Carmo Barcelos Correia(2) \\ e Gener Tadeu Pereira ${ }^{(3)}$
}

(1)Universidade Estadual Paulista (Unesp), Fac. de Ciências Agrárias e Veterinárias (FCAV), Dep. de Produção Vegetal, Via de Acesso Prof. Paulo Donato Castellane s/no, CEP 14884-900 Jaboticabal, SP. E-mail: montecar@fcav.unesp.br, ccbcamargo@uol.com.br (2)Unesp, FCAV, Dep. de Fitossanidade. E-mail: antoniac@fcav.unesp.br (3)Unesp, FCAV, Dep. de Ciências Exatas. E-mail: genertp@fcav.unesp.br

Resumo - O objetivo deste trabalho foi avaliar o crescimento e a esporulação de dois isolados (JAB 02 e JAB 45) de Verticillium lecanii (Zimm.) Viégas, submetidos a diferentes valores de $\mathrm{pH}(4,5,6,7,8,9)$ inicial do meio de cultivo sólido, de temperatura $\left(19,22,25,28\right.$ e $\left.31^{\circ} \mathrm{C}\right)$ e fotoperíodo $(0,12$ e 24 horas). Após 20 dias de cultivo, não se observou efeito do $\mathrm{pH}$ inicial do meio no crescimento e esporulação dos isolados, exceto no pH 4,0 que reduziu o crescimento. A ausência de iluminação proporcionou a melhor condição para o crescimento de JAB 02 (32,70 mm) e JAB 45 (32,67 mm). Não houve efeito do fotoperíodo na produção de esporos. As melhores temperaturas para o crescimento de JAB 02 foram 19,22 e $25^{\circ} \mathrm{C}$ e para JAB 45 foram $19 \mathrm{e} 25^{\circ} \mathrm{C}$. Este último isolado apresentou melhor esporulação a 19 e $22^{\circ} \mathrm{C}$, mas a temperatura não influenciou a esporulação de JAB 02 . Não houve desenvolvimento dos isolados a $31^{\circ} \mathrm{C}$

Termos para indexação: fungo entomopatogênico, $\mathrm{pH}$, temperatura, fotoperíodo, controle de pragas.

\section{Growth and sporulation of Verticillium lecanii isolates under different environmental conditions}

\begin{abstract}
This research aimed to evaluate the growth and sporulation of two Verticillium lecanii (Zimm.) Viégas isolates cultivated on solid medium with different initial $\mathrm{pH}$ values $(4,5,6,7,8$ and 9$)$, exposed to different temperatures $\left(19,22,25,28\right.$ and $\left.31^{\circ} \mathrm{C}\right)$ and photoperiods $(0,12$ and 24 hours). There was no substantial effect of the initial $\mathrm{pH}$ values on growth and sporulation of both isolates twenty days after inoculation, except at $\mathrm{pH} 4.0$ in which the growth was significantly reduced. Absence of light promoted the best growth of JAB $02(32.70 \mathrm{~mm})$ and JAB $45(32.67 \mathrm{~mm})$ isolates but there was no effect of photoperiod on sporulation. The best temperatures for growth of JAB 02 were 19,22 and $25^{\circ} \mathrm{C}$ and for $\mathrm{JAB} 45,19$ and $25^{\circ} \mathrm{C}$. The latter isolate sporulated most at 19 and $22^{\circ} \mathrm{C}$ and there was no effect of temperature on sporulation of JAB 02 . None of the isolates developed at $31^{\circ} \mathrm{C}$.
\end{abstract}

Index terms: entomopathogenic fungi, $\mathrm{pH}$, temperature, photoperiod, pest control.

\section{Introdução}

O fungo Verticillium lecanii (Zimm.) Viégas é patógeno de várias espécies de insetos (Ferron, 1981 Alves, 1998) e um dos mais promissores no controle biológico de pragas na agricultura (Lecuona \& Riba 1991). Entretanto, para a formulação de bioinseticida à base desse fungo é necessária a obtenção de boa produção massal com abundante esporulação.

Um fator importante na produção massal de um fungo é a seleção de um meio padrão para seu cultivo e a determinação das condições adequadas que permitam bom crescimento com alta esporulação (Khalil et al.
1985). A seleção de um meio padrão pressupõe o conhecimento das condições nutricionais ideais para o cultivo do fungo. Barbosa et al. (2002) avaliaram diferentes meios de cultura, fontes de $\mathrm{C}$, de $\mathrm{N}$ e relações $\mathrm{C}: \mathrm{N}$ no desenvolvimento dos isolados JAB 02 e JAB 45 de V. lecanii. Wenzel (2002) analisou diversas fontes de $\mathrm{P}$ e a influência de relações C:P e C:N:P no crescimento e esporulação dos mesmos isolados.

Na determinação das condições ambientais necessárias para otimizar a produção do fungo, fatores como $\mathrm{pH}$, iluminação e temperatura podem se tornar limitantes. A temperatura tem influência significativa no desenvolvimento de V. lecanii (Verhaar \& Hijwegen, 1993; 
Hanlon et al., 1994). As faixas térmicas ideais para o crescimento micelial, esporulação e germinação de conídios do fungo são de $23-28,23-25$ e $15-32^{\circ} \mathrm{C}$, respectivamente (Li et al., 1991). O cultivo de $V$. lecani em meio líquido alterou o $\mathrm{pH}$ do meio (Galani, 1987), e o pH 7 favoreceu a produção do fungo (Hanlon et al., 1994). O regime de iluminação contínua proporcionou maior crescimento e esporulação de $V$. fungicola, em comparação com a ausência de iluminação (Coetzee \& Eicker, 1991).

Verticillium lecanii pode ser considerado um complexo de espécies que abrange isolados com grande variação nos aspectos morfológicos e bioquímicos (Steenberg \& Humber, 1999). Estudos que visam determinar as condições ideais de cultivo de isolados obtidos no Brasil podem viabilizar a produção massal do fungo no país.

O objetivo deste trabalho foi avaliar o efeito de diferentes valores de $\mathrm{pH}$ do meio de cultivo, de temperatura e fotoperíodo no crescimento e esporulação de dois isolados de $V$. lecanii.

\section{Material e Métodos}

Foram utilizados os isolados JAB 02 e JAB 45 de Verticillium lecanii, mantidos na coleção do Laboratório de Microbiologia da FCAV/Unesp, obtidos da Cochonilha Verde Coccus viridis Green (Hemiptera: Coccidae), coletada em pomares de laranja (Citrus sinenis Osbeck), nos municípios paulistas de Ubirajara e São Carlos, respectivamente. Os isolados foram cultivados em tubos de ensaio contendo meio de batatadextrose-ágar (BDA), por dez dias a $27 \pm 0,5^{\circ} \mathrm{C}$ e fotófase de 14 horas, e as culturas estocadas em refrigerador a cerca de $4^{\circ} \mathrm{C}$

Alíquotas do fungo, retiradas da cultura estoque, foram inoculadas em placas de Petri contendo meio BDA e incubadas a $27 \pm 0,5^{\circ} \mathrm{C}$, com fotófase de 14 horas, por 20 dias. Culturas assim rejuvenescidas serviram de inóculo para os ensaios.

O cultivo foi realizado em placas de Petri de $12 \times 80 \mathrm{~mm}$, contendo $15 \mathrm{~mL}$ de meio BDA para JAB 02 e meio completo de Pontecorvo para JAB 45 (Barbosa et al., 2002). A inoculação foi efetuada com agulha de níquel-cromo mediante simples picada, transferindo-se esporos e fragmentos de micélio para o ponto central das placas. Após inoculação, o fungo foi incubado em estufa a $27 \pm 0,5^{\circ} \mathrm{C}$, por 20 dias, no escuro.
A avaliação do crescimento radial da colônia foi realizada com régua milimétrica, medindo-se, a cada dois dias, durante 20 dias, dois diâmetros previamente marcados na face externa do fundo de cada placa de Petri. Foram feitas seis repetições por tratamento, considerando-se cada placa uma repetição. A produção de esporos foi avaliada no $20^{\circ}$ dia de incubação. Para tanto, escolheram-se, ao acaso, três placas de cada tratamento (três repetições por tratamento), retirando-se de cada uma, com auxílio de um furador de rolha metálico, três discos de $8 \mathrm{~mm}$ de diâmetro, sendo um da região central, outro da região mediana e outro da região periférica das colônias. Os discos foram transferidos individualmente para tubos de ensaio contendo $10 \mathrm{~mL}$ de uma mistura (1:1) de solução salina $(\mathrm{NaCl}$ a $0,89 \%$ p/v) e solução de Tween 80 (0,1\% v/v). Após vigorosa agitação em agitador elétrico de tubos, extraíram-se os conídios e procedeu-se à contagem em câmara de Neubauer.

Três ensaios foram realizados para avaliar o crescimento e a esporulação do fungo em relação aos fatores ambientais de cultivo. No primeiro, analisou-se o efeito do $\mathrm{pH}$ inicial do meio, cujos valores, 4, 5, 6, 7, 8 e 9, foram obtidos mediante adição ao meio de cultivo de uma solução 0,5 ou $1,0 \mathrm{~N}$ de $\mathrm{NaOH}$ ou $\mathrm{HCl}$, quando se pretendeu aumentar ou diminuir o $\mathrm{pH}$, respectivamente. Os valores de $\mathrm{pH}$ foram medidos com auxílio de um potenciômetro.

No segundo ensaio, avaliou-se a influência do fotoperíodo. No regime de iluminação contínua, três lâmpadas fluorescentes de $40 \mathrm{~W}$ foram posicionadas $50 \mathrm{~cm}$ acima das placas com fungos, expondo-as aos raios luminosos. Na iluminação alternada, as placas foram envolvidas, a cada 12 horas, em um tecido negro de algodão e, nas 12 horas subseqüentes, foram mantidas sob a condição anterior de iluminação. A ausência de iluminação foi obtida pelo envolvimento contínuo das placas com fungo em tecido negro. O ensaio foi conduzido em uma sala ambiente, com temperatura mantida entre 25 e $28^{\circ} \mathrm{C}$.

No terceiro ensaio, avaliou-se o efeito das temperaturas de $19,22,25,28$ e $31^{\circ} \mathrm{C}$ no desenvolvimento dos isolados. As placas com fungo foram acondicionadas em câmaras climatizadas BOD previamente aferidas com as temperaturas ensaiadas.

Os ensaios foram conduzidos segundo o delineamento inteiramente casualizado. Os dados de crescimento foram analisados por meio de regressão linear, seguida de teste de paralelismo (teste t) e coincidência (teste F) 
entre as retas (Dixon \& Massey Junior, 1969). Os valores obtidos na produção de esporos foram submetidos à análise de variância pelo teste $\mathrm{F}$ a $1 \%$ de probabilidade e as médias, comparadas pelo teste de Tukey a $5 \%$ de probabilidade.

\section{Resultados e Discussão}

$\mathrm{O}$ crescimento dos isolados no meio com $\mathrm{pH}$ inicia 4,0 diferiu significativamente $(p<0,01$, pelos testes te $F$ entre as retas) dos demais meios, os quais, por sua vez, não diferiram significativamente entre si, para ambos os isolados, até mesmo no valor de $\mathrm{pH} 9,0$, considerado crítico ao desenvolvimento de fungos (Figura 1). Quanto à esporulação, não houve diferença significativa ( $>>0,05)$ entre os valores de $\mathrm{pH}$ avaliados (Tabela 1).

V. lecanii produziu a maior biomassa $\left(14,82 \mathrm{mg} \mathrm{mL}^{-1}\right)$ em meio de cultura com pH 7,0 (Hanlon et al., 1994). Segundo estes autores, raramente o pH do meio se mantém inalterado durante o crescimento, por causa da degradação de proteínas que produzem compostos alcalinos e da fermentação de carboidratos. Após cultivar $V$. lecani em meios líquidos com 10 valores de $\mathrm{pH}$ iniciais varian-
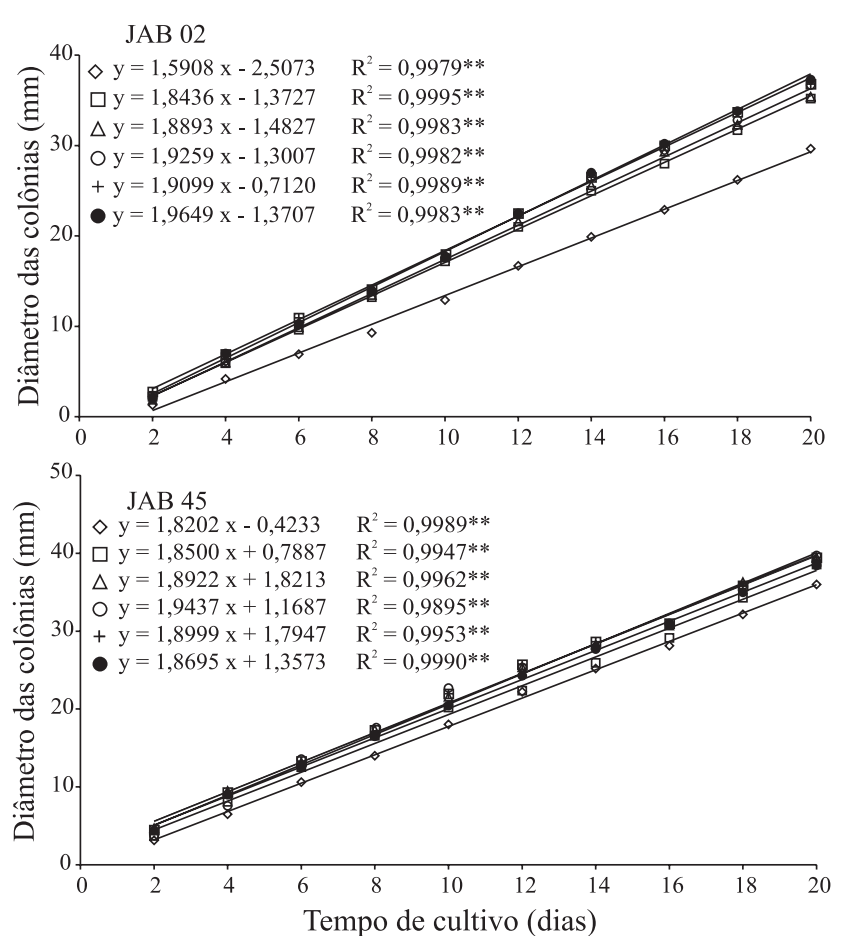

Figura 1. Crescimento radial de Verticillium lecanii cultivado a pH $4(\diamond), 5(\square), 6(\triangle), 7(0), 8(+)$ e $9(\bullet)$. **Significativo a $1 \%$ de probabilidade pelo teste $\mathrm{F}$. do entre 4 e 8,5, Galani (1987) verificou que, nos meios com $\mathrm{pH}$ inicial mais alto, ocorreu queda acentuada de alcalinidade, mas de modo geral o $\mathrm{pH}$ aumentou, ficando na faixa entre 5 e 7 após 96 horas de cultivo. Magalhães et al. (1994) observaram que a variação do pH estava relacionada com a concentração inicial de conídios de Metarhizium anisopliae inoculados em meio líquido, indicando a utilização de fonte nitrogenada pelo fungo. Segundo Hallsworth \& Magan (1996), a melhor faixa de $\mathrm{pH}$ para o crescimento de $M$. anisopliae, Beauveria bassiana e Paecilomyces farinosus situase entre 5,0 e 8,0. Os resultados indicam que não houve grande influência do $\mathrm{pH}$ inicial sobre o fungo, que foi capaz de superar possíveis restrições impostas pelos diferentes $\mathrm{pH}$ do meio, apresentando igual desempenho em todos os tratamentos, exceto no $\mathrm{pH}$ mais ácido.

Ambos os isolados apresentaram melhor crescimento em ausência de luz com diâmetro de colônias significativamente maiores $(\mathrm{p}<0,01$, pelos testes $t$ e $F$ entre as retas) do que quando mantidos na presença dos outros dois tratamentos. A fotófase de 12 horas ocasionou ligeira redução do crescimento. A maior redução foi observada com o isolado JAB 45 submetido à presença constante de luz (Figura 2). Não houve efeito da iluminação na esporulação de ambos os isolados (Tabela 1).

Tabela 1. Esporulação dos isolados JAB 02 e JAB 45 de Verticillium lecanii ( $\mathrm{n}^{\mathrm{o}}$ de conídios $\times 10^{4} \mathrm{~mL}^{-1}$ ) no vigésimo dia de cultivo em meios com diversos valores de $\mathrm{pH}$ inicial, diferentes fotoperíodos e temperaturas ${ }^{(1)}$.

\begin{tabular}{lrc}
\hline Fator físico & JAB 02 & JAB 45 \\
\hline pH inicial do meio & & \\
4 & $119,10 \mathrm{a}$ & $64,17 \mathrm{a}$ \\
5 & $48,27 \mathrm{a}$ & $124,33 \mathrm{a}$ \\
6 & $109,38 \mathrm{a}$ & $120,56 \mathrm{a}$ \\
7 & $77,78 \mathrm{a}$ & $101,39 \mathrm{a}$ \\
8 & $107,29 \mathrm{a}$ & $128,34 \mathrm{a}$ \\
9 & $61,46 \mathrm{a}$ & $105,00 \mathrm{a}$ \\
\hline CV $(\%)$ & 48,83 & 48,24 \\
\hline Fotoperíodo (h) & & \\
24 & $19,72 \mathrm{a}$ & $25,55 \mathrm{a}$ \\
12 & $17,22 \mathrm{a}$ & $23,33 \mathrm{a}$ \\
0 & $36,39 \mathrm{a}$ & $117,22 \mathrm{a}$ \\
\hline $\mathrm{CV}(\%)$ & 91,52 & 74,69 \\
\hline Temperatura $\left({ }^{\circ} \mathrm{C}\right)$ & & \\
19 & $9,17 \mathrm{a}$ & $180,83 \mathrm{ab}$ \\
22 & $78,33 \mathrm{a}$ & $304,17 \mathrm{a}$ \\
25 & $73,05 \mathrm{a}$ & $28,61 \mathrm{~b}$ \\
28 & $102,50 \mathrm{a}$ & $21,67 \mathrm{~b}$ \\
\hline CV $(\%)$ & 65,16 & 64,51
\end{tabular}

${ }^{(1)}$ Médias seguidas pela mesma letra, na coluna, não diferem entre si pelo teste de Tukey a $5 \%$ de probabilidade. 
V. fungicola cresceu e esporulou melhor em regime de luz contínua (Coetzee \& Eicker, 1991). Segundo Nahas \& Arai (1987), o melhor crescimento e esporulação de $B$. bassiana foi observado em fotófase de 12 horas. Monteiro (1988) obteve melhor crescimento de M. anisopliae, B. bassiana e Paecilomyces marquandii em ausência de luz, mas a melhor esporulação foi obtida em fotófase de 12 horas, exceto para $P$. marquandii, cuja produção de esporos foi favorecida pela ausência de iluminação. $\mathrm{O}$ crescimento reduzido em presença de luz é uma característica constante em fungos (Cochrane, 1958) e a alternância entre períodos de presença e ausência de luz favorece determinadas reações químicas que contribuem para o desenvolvimento de fungos (Lilly \& Barnett, 1951). Contudo, a fotófase de 12 horas não proporcionou melhor desempenho dos isolados avaliados neste trabalho.

$\mathrm{O}$ crescimento de ambos os isolados foi favorecido pelas temperaturas de 19,22 e $25^{\circ} \mathrm{C}$ que apresentaram diferença significativa ( $\mathrm{p}<0,01$, pelos testes t e $\mathrm{F}$ entre as retas) em relação ao crescimento obtido na temperatura de $28^{\circ} \mathrm{C}$. Contudo, em relação ao JAB 45 , o de sempenho a partir do $18 \mathrm{o}$ dia de cultivo a $22^{\circ} \mathrm{C}$ foi inferior ao verificado a $19^{\circ} \mathrm{C}$ (Figura 3). Não se observou
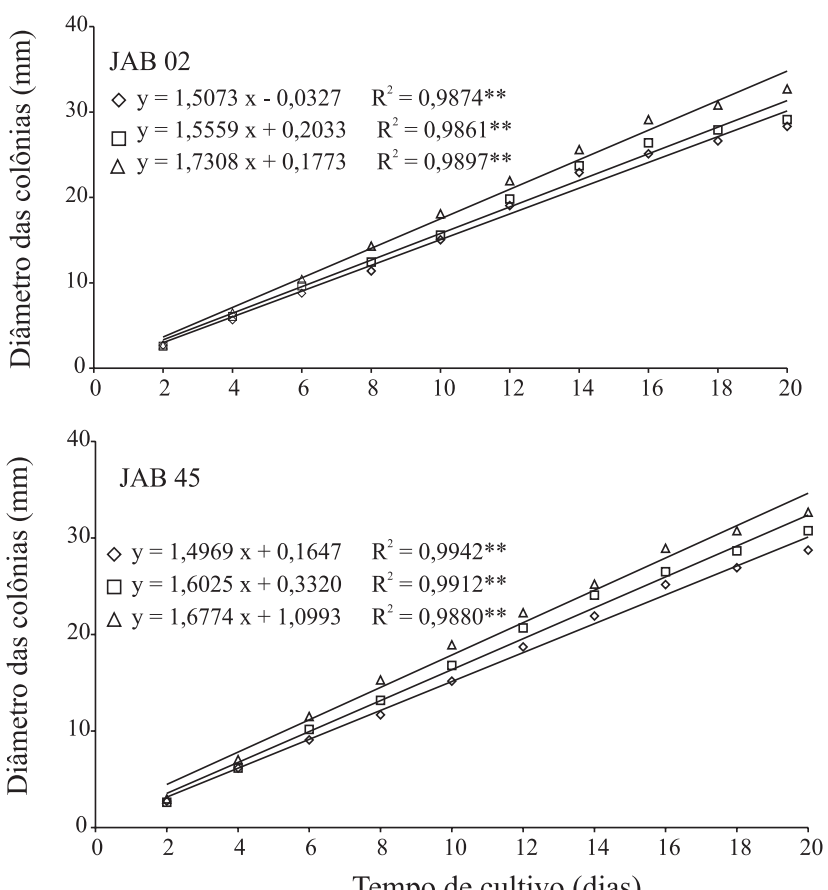

Figura 2. Crescimento radial de Verticillium lecanii cultivado por $24(\diamond), 12(\square)$ e 0 hora $(\triangle)$. **Significativo a $1 \%$ de probabilidade pelo teste $\mathrm{F}$. efeito da temperatura na esporulação do isolado $\mathrm{JAB} 02$, embora o valor verificado a $28^{\circ} \mathrm{C}$ tenha sido 11 vezes superior ao encontrado a $19^{\circ} \mathrm{C}$. Possivelmente, a redução do crescimento deste isolado na temperatura de $28^{\circ} \mathrm{C}$ provocou maior empenho em aumentar a esporulação como tentativa de garantir a sobrevivência do fungo no ambiente. As maiores esporulações de JAB 45 ocorreram a 19 e $22^{\circ} \mathrm{C}$, embora apenas a ocorrida a $22^{\circ} \mathrm{C}$ tenha diferido significativamente das verificadas a 25 e $28^{\circ} \mathrm{C}$ (Tabela 1 ). Não houve desenvolvimento dos isolados quando cultivados a $31{ }^{\circ} \mathrm{C}$

Os dados encontrados sugerem que a faixa térmica mais favorável para o desenvolvimento de $V$. lecanii em laboratório situa-se entre 20 e $25^{\circ} \mathrm{C}$. De acordo com Li et al. (1991), o melhor crescimento e a melhor esporulação do fungo ocorreram, respectivamente, a $23-28^{\circ} \mathrm{C}$ e $23-25^{\circ} \mathrm{C}$. A melhor produção de biomassa ocorreu a $24^{\circ} \mathrm{C}$ (Hanlon et al., 1994). Segundo Verhaar \& Hijwegen (1993), V. lecanii não produziu conídios quando cultivado a $30^{\circ} \mathrm{C}$ por dez dias e a maior esporulação foi obtida a $25^{\circ} \mathrm{C}$.

Os resultados do presente trabalho são semelhantes aos obtidos por estes autores e indicam que a faixa térmica mais favorável ao desenvolvimento do fungo si-
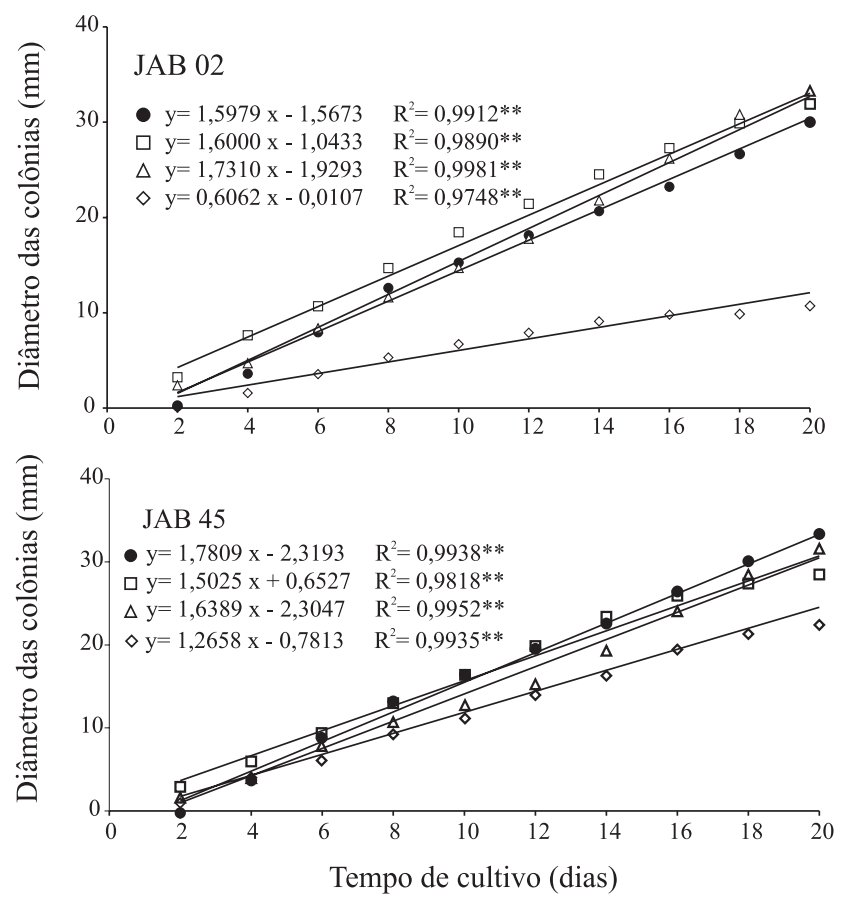

Figura 3. Crescimento radial de Verticillium lecanii cultivado a $19(\bigcirc), 22(\square), 25(\triangle)$ e $28^{\circ} \mathrm{C}(\diamond)$. **Significativo a $1 \%$ de probabilidade pelo teste $\mathrm{F}$. 
tua-se entre 19 e $25^{\circ} \mathrm{C}$. Houve redução do crescimento de ambos os isolados na temperatura de $28^{\circ} \mathrm{C}$. Com relação à esporulação, observa-se diferença na preferência entre os isolados, tendo JAB 45 esporulado melhor nas temperaturas mais baixas, enquanto $\mathrm{JAB} 02$ tendeu a aumentar a produção de conídios nas temperaturas mais altas. Esta diferença na preferência pode ser atribuída à variabilidade genética existente na espécie, com grande variação nos aspectos morfológicos e bioquímicos entre os isolados, como referido por Steenberg \& Humber (1999).

\section{Conclusões}

1. $\mathrm{O}$ pH inicial do meio de cultivo não afeta severamente o desempenho dos isolados, mas o $\mathrm{pH} 4,0$ reduz o crescimento.

2. O isolados crescem melhor em ausência de luz e o fotoperíodo não afeta a esporulação.

3. A faixa térmica favorável para o crescimento e esporulação dos isolados situa-se entre 19 e $25^{\circ} \mathrm{C}$.

\section{Agradecimentos}

À Coordenadoria de Aperfeiçoamento de Pessoal de Ensino Superior (Capes) pela concessão da Bolsa de Mestrado ao segundo autor.

\section{Referências}

ALVES, S.B. Fungos entomopatogênicos. In: ALVES, S.B. (Ed.) Controle microbiano de insetos. 2.ed. Piracicaba: Fealq, 1998. p.289-382.

BARBOSA, C.C.; MONTEIRO, A.C.; CORREIA, A. do C.B. PEREIRA, GT Crescimento e esporulação de isolados de Verticillium lecanii sob diferentes condições nutricionais. Pesquisa Agropecuária Brasileira, v.24, p.375-381, 2002

COETZEE, J.C.; EICKER, A. The effect of nutritional and environmental factors on the growth and sporulation of a Southern African isolate of Verticillium fungicola. In: INTERNATIONAL CONGRESS ON THE SCIENCE AND CULTIVATION OF EDIBLE FUNGI, 13., 1991, Dublin. Proceedings. Dublin: Balkema, 1991. v.2, p.417-424.

COCHRANE, V.W. Physiology of fungi. New York: J. Wiley, 1958. $524 \mathrm{p}$

DIXON, W.J.; MASSEY JUNIOR, F.J. Introduction to statistica analysis. 3rd ed. Tokyo: McGraw-Hill Kogakusha, 1969. 638p.
FERRON, P. Pest control by the fungi Beauveria bassiana. In: BURGES, H.D. (Ed.). Microbial control of pest and plant diseases. London: Academic, 1981. p.465-482.

GALANI, G. Cultivarea unor specii de ciuperci entomopatogene in medii lichide cu valori de $\mathrm{pH}$ initial diferite. Analele Institutului de Cercetari Pentru Protectia Plantelor, v.21, p.45-54, 1987.

HALLSWORTH, J.E.; MAGAN, N. Culture age, temperature and $\mathrm{pH}$ affect the polyol and trehalose contents of fungal propagules. , v.62, p.2435-2442, 1996.

HANLON, G.W.; KOOLOOBANDI, A.; HUTT, A.J. Microbial metabolism of 2-arypropionic acid: effect of environmental on the metabolism of ibuprofen by Verticillium lecanii. Journal of Applied Bacteriology, v.76, p.442-447, 1994.

KHALIL, S.K.; SHAH, M.A.; NAEEM, M. Laboratory studies on the compatibility of the entomopathogenic fungus Verticillium lecanii with certain pesticides. Agriculture, Ecosystems and Environment, v.13, p.329-334, 1985 .

LECUONA, R.E.; RIBA, G. Primeras etapas del ciclo de desarrollo de hongos entomopatógenos. Pergamino: Instituto Nacional de Tecnología Agropecuaria, 1991. 30p. (Boletin de Divulgación Tecnológica, 87).

LI, G.; YUHUA, Y.; LIYING, W. Influence of temperature and nutrition on growth of the entomopathogenic fungus, Verticillium lecanii (Beijing strain). Chinese Journal of Biological Control, v.7, p.115-119, 1991.

LILLY, V.G.; BARNETT, H.L. Physiology of the fungi. New York: MacGraw-Hill Book, 1951. 464p.

MAGALHÃES, B.P.; DIAS, J.M.C.S.; FERREIRA, C.M. Mycelial production of Metarhizium anisopliae in liquide culture using different sources of carbon and nitrogen. Revista de Microbiologia, v.25, p.181-187, 1994.

MONTEIRO, A.C. Aspectos fisioecológicos de isolados de fungos entomopatogênicos obtidos na região amazônica (Manaus) 1988. 233p. Tese (Doutorado) - Universidade Federal de São Carlos, São Carlos.

NAHAS, E.; ARAI, N.N.S. Crescimento e esporulação de Beauveria bassiana em vários meios e condições de cultivo. Revista de Microbiologia, v.18, p.77-82, 1987.

STEENBERG, T.; HUMBER, R.A. Entomopathogenic potencial of Verticillium lecanii and Acremonium species (Deuteromycotina: Hyphomycetes). Journal of Invertebrate Pathology, v.73, p.309314, 1999

VERHAAR, M.A.; HIJWEGEN, T. Efficient production of phialoconidia of Verticillium lecanii for biocontrol of cucumber powdery mildew, Spharotheca fuliginea. Netherlands Journal of Plant Pathology, v.99, p.101-103, 1993.

WENZEL, I.M. Fatores nutricionais e produção em massa de Verticillium lecanii em meios naturais líquidos e sólidos. 2002. 70p. Dissertação (Mestrado) - Universidade Estadual Paulista, Jaboticabal.

$\overline{\text { Recebido em } 21 \text { de novembro de } 2003 \text { e aprovado em } 29 \text { de março de } 2004}$ 